\title{
Magnetic-field instability of Majorana modes in multiband semiconductor wires
}

\author{
Jong Soo Lim, ${ }^{1}$ Llorenç Serra, ${ }^{1,2}$ Rosa López, ${ }^{1,2}$ and Ramón Aguado ${ }^{3}$ \\ ${ }^{1}$ Institut de Física Interdisciplinària i de Sistemes Complexos IFISC (CSIC-UIB), E-07122 Palma de Mallorca, Spain \\ ${ }^{2}$ Departament de Física, Universitat de les Illes Balears, E-07122 Palma de Mallorca, Spain \\ ${ }^{3}$ Teoría y Simulación de Materiales, Instituto de Ciencia de Materiales de Madrid, ICMM-CSIC Cantoblanco, E-28049 Madrid, Spain
}

(Received 15 February 2012; revised manuscript received 11 May 2012; published 10 September 2012)

\begin{abstract}
We investigate the occurrence of Majorana modes in semiconductor quantum wires in close proximity with a superconductor and when both Rashba interaction and magnetic field are present. We consider long, but finite, multiband wires (namely, planar wires with dimensions $L_{x} \gg L_{y}$ ). Our results demonstrate that interband mixing coming from the Rashba spin-orbit term hybridizes Majorana pairs originating from different transverse modes while simultaneously closing the effective gap. Consequently, multiple Majorana modes do not coexist in general. On the contrary, Majorana physics is robust provided that only one single transverse mode contributes to a Majorana pair. Finally, we analyze the robustness of Majorana physics with respect to magnetic orbital effects.
\end{abstract}

Introduction. Matter and its charge conjugate counterpart obey the relativistic Dirac equation with positive and negative energies, respectively. ${ }^{1}$ Ettore Majorana proved in 1937 that these solutions exist for which particle and antiparticle are the same entity, a Majorana fermion. ${ }^{2}$ Majorana fermions were first proposed in the context of particle physics to describe neutrinos $^{3}$ and, more recently, the Majorana search has been revived $^{4}$ in the condensed-matter ${ }^{5-16}$ and atomic physics ${ }^{17-20}$ communities. Aside from a fundamental interest in finding Majorana fermions, part of the excitement comes from the non-Abelian braiding statistics appearing when these particles are localized near a vortex or a domain wall, which could be useful for topological quantum computation. ${ }^{21}$

The most recent and promising proposal for engineering Majorana quasiparticles is based on semiconductor nanowires (NWs) put in close proximity with a superconductor when both spin-orbit (SO) interaction and magnetic field are present. The main advantages of these systems are that they exhibit giant Zeeman splittings due to the huge $g$ factor and that conventional $s$-wave superconductivity can be proximity induced. ${ }^{22-24}$ Majorana modes are quasiparticle excitations that emerge when the Kramers degeneracy of the electron-hole pairs is lifted because of the presence of both Rashba SO and magnetic field. Nontrivial phases that correspond to the occurrence of Majorana physics have been predicted to survive even in multimode NWs provided that the number of filled subbands is odd. ${ }^{25-29}$ In this manner, trivial and nontrivial topological phases can be alternated by tuning either the chemical potential or the magnetic field. Nevertheless, this scenario of alternating robust (nontrivial) and fragile (trivial) phases is based on topological arguments strictly valid for infinitely long strips that are characterized by the $Z_{2}$ invariant, such as the Majorana number. ${ }^{30}$ In order to unambiguously predict the occurrence of Majorana modes in realistic systems, one needs to investigate finite multimode nanowires. Such studies have been previously attempted in a two-band model and for tight-binding Hamiltonians. ${ }^{25-27,31}$ Here, we perform exact numerical diagonalization of the Hamiltonian. Our main finding indicates that multiple Majorana modes in finite multiband nanowires cannot coexist due to the strong hybridization caused by the Rashba spin-orbit interaction. The importance of the Rashba coupling term in planar wires has been emphasized by some of us. ${ }^{32-34}$ We observe the formation of a single Majorana pair at low magnetic fields only. Furthermore, we investigate the magnetic orbital effects in these systems and we show that the single Majorana pair regime survives solely if the magnetic field is in the plane of the wire.

The model. We consider a semiconductor nanowire with a strong Rashba SO and proximity-induced pairing in the presence of a magnetic field $B$ with a given orientation as sketched in Fig. 1. The model Hamiltonian can be written as $\mathcal{H}_{N W}=\mathcal{H}_{S P}+\mathcal{H}_{Z}+\mathcal{H}_{S O}$, where

$$
\begin{gathered}
\mathcal{H}_{S P}=\frac{\Pi_{x}^{2}+\Pi_{y}^{2}}{2 m^{*}}-\mu+V\left(x ; L_{x}\right)+V\left(y ; L_{y}\right), \\
\mathcal{H}_{Z}=\frac{1}{2} g \mu_{B} \vec{B} \cdot \vec{\sigma}, \\
\mathcal{H}_{S O}=\frac{\alpha_{R}}{2}(\vec{\sigma} \times \vec{\Pi}) \cdot \overrightarrow{\mathcal{E}},
\end{gathered}
$$

with $m^{*}$ the effective mass, $\Pi_{i}=p_{i}+(e / c) A_{i}$ the canonical momentum ( $e$ is the electron's charge and $c$ is the speed of light), and $\vec{A}$ the vector potential. $\mathcal{H}_{S P}$ is the single particle energy, referred to the chemical potential $\mu$ with a hard-wall confinement in both $x$ and $y$ directions given by $V(a ; L)=0$ for $0<a<L$ and $V(a ; L)=\infty$ otherwise. $\mathcal{H}_{Z}$ corresponds to the Zeeman term with $\Delta_{B} \equiv g \mu_{B} B / 2 . \mathcal{H}_{S O}$ is the Rashba SO Hamiltonian. The constant vector $\overrightarrow{\mathcal{E}}$ in $\mathcal{H}_{S O}$ corresponds to the effective electric field due to confinement. In our planar geometry (Fig. 1), typical of Rashba systems, the strongest confinement occurs along the $z$ axis and, therefore, $\mathcal{H}_{S O} \approx$ $\alpha / \hbar\left(\sigma_{x} \Pi_{y}-\sigma_{y} \Pi_{x}\right)$ with $\alpha=\alpha_{R} \mathcal{E}_{z} / 2$. In a convenient gauge $\left(\vec{A}=-B_{z} y \hat{u}_{x}\right) \Pi_{x}=p_{x}-e y B_{z} / c$ and $\Pi_{y}=p_{y}$. Notice then that both $\mathcal{H}_{S P}$ and $\mathcal{H}_{S O}$ are modified by the magnetic orbital effects, represented by the magnetic length $\ell_{z}=\sqrt{\hbar c / e B_{z}}$. More specifically, in the case of the Rashba Hamiltonian we have

$$
\mathcal{H}_{S O}=\frac{\alpha_{x}}{\hbar} p_{x} \sigma_{y}-\frac{\alpha_{y}}{\hbar} p_{y} \sigma_{x}-\frac{\alpha_{x}}{\hbar} \frac{y \sigma_{y}}{\ell_{z}^{2}},
$$

where the distinction between $\alpha_{x}$ and $\alpha_{y}$ is introduced for later convenience. Clearly, in Rashba NWs $\alpha_{x}=\alpha_{y}=\alpha$; however, situations with $\alpha_{y} \neq \alpha_{x}$, including the case $\alpha_{y}=0$ have been 


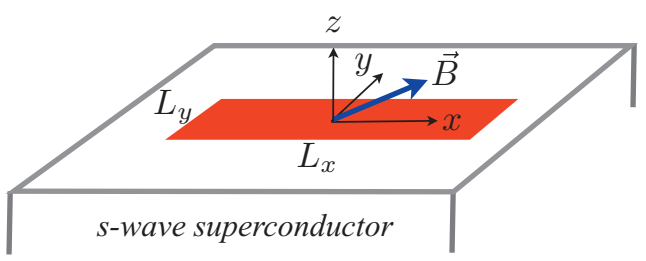

FIG. 1. (Color online) Sketch of a finite nanowire of dimensions $\left(L_{x}, L_{y}\right)$ in close proximity with an s-wave superconductor and in presence of a tilted magnetic field.

theoretically proposed in NWs without spin-orbit interaction using inhomogeneous fields $B(x)$ or spatially modulated $g$ factors. ${ }^{35}$ In general, Eq. (4) mixes different quantum well subbands in $x$ (through the $p_{x}$ operator) and in $y\left(p_{y}\right.$ and $y$ operators). Since $L_{x} \gg L_{y}$ the relevant mixing contribution is $\alpha_{y} p_{y} \sigma_{x}$, which we termed as Rashba mixing. Finally, we stress that orbital effects modify the SO coupling, including the Rashba mixing. As shown below, they dramatically alter the topological phases of multiband NWs.

The second-quantized Hamiltonian can be written as $\mathcal{H}_{N W} \rightarrow \sum_{n, n^{\prime}} \sum_{\sigma, \sigma^{\prime}}\left\langle n \sigma\left|\mathcal{H}_{N W}\right| n^{\prime} \sigma^{\prime}\right\rangle c_{n \sigma}^{\dagger} c_{n^{\prime} \sigma^{\prime}}$, where $n \equiv$ $\left\{n_{x}, n_{y}\right\}$ are the quantum numbers associated with the transverse modes due to confinement and $\sigma=\uparrow, \downarrow$ denotes the spin. When the NW is proximity coupled to an ordinary $s$-wave superconductor the BCS-Hamiltonian is taken into account, $\mathcal{H}_{S C}=\sum_{n}\left[\Delta c_{n \uparrow}^{\dagger} c_{n \downarrow}^{\dagger}+\right.$ H.c. $]$. One then obtains the low-energy physics of this system in a Bogoliubov-de Gennes description. In matrix form, $\mathcal{H}_{B d G}=\frac{1}{2} \sum_{n, n^{\prime}} \Psi_{n}^{\dagger} \mathcal{H}_{B d G}^{\left(n n^{\prime}\right)} \Psi_{n^{\prime}}$, where $\Psi_{n}=\left(c_{n \uparrow}^{\dagger}, c_{n \downarrow}^{\dagger}, c_{n \downarrow},-c_{n \uparrow}\right)^{\dagger}$ and

$$
\mathcal{H}_{B d G}^{\left(n n^{\prime}\right)}=\left(\begin{array}{cc}
\mathcal{H}_{N W}^{n n^{\prime}} & \Delta \\
\Delta^{*} & i \sigma_{y}\left[\mathcal{H}_{N W}^{n n^{\prime}}\right]^{*}{ }_{i \sigma_{y}}
\end{array}\right) .
$$

The results shown below are obtained by exact numerical diagonalization of Eq. (5) and confirm the emergence of NW gapped low-energy eigenstates for some range of parameters.

The existence of gapped zero-energy modes is the signature of Majorana physics. Let us label positive-energy states $E_{I}$ by an index $I=1,2, \ldots$ in increasing energy order. Analogously, negative-energy states in decreasing energy order are labeled by $I=-1,-2, \ldots$ In the Dirac picture of fermions, positiveenergy states are particle states, while negative ones are their conjugated or antiparticle ones. Clearly, when $\left|E_{I}\right|$ is sizable nonzero $|I\rangle$ and $|-I\rangle$ are different stationary eigenstates of $\mathcal{H}_{B d G}$. We can now form the two combinations

$$
\begin{aligned}
\left|\gamma_{a}^{(I)}\right\rangle & =\frac{1}{\sqrt{2}}(|I\rangle+|-I\rangle), \\
\left|\gamma_{b}^{(I)}\right\rangle & =\frac{i}{\sqrt{2}}(|I\rangle-|-I\rangle) .
\end{aligned}
$$

These are the Majorana states that, in general, are not eigenstates of $\mathcal{H}_{B d G}$ [see Eq. (4)] unless $E_{I} \approx E_{-I} \approx 0$. These states correspond to zero modes separated by a sizable energy gap from the rest of the spectrum. In the following we will carefully investigate the robustness of this behavior in finite samples and the role of the Rashba mixing term.

Occurrence of Majorana modes in finite nanowires. It is estimated that in infinite strictly one-dimensional (1D) quantum wires the occurrence of a Majorana pair requires sufficiently

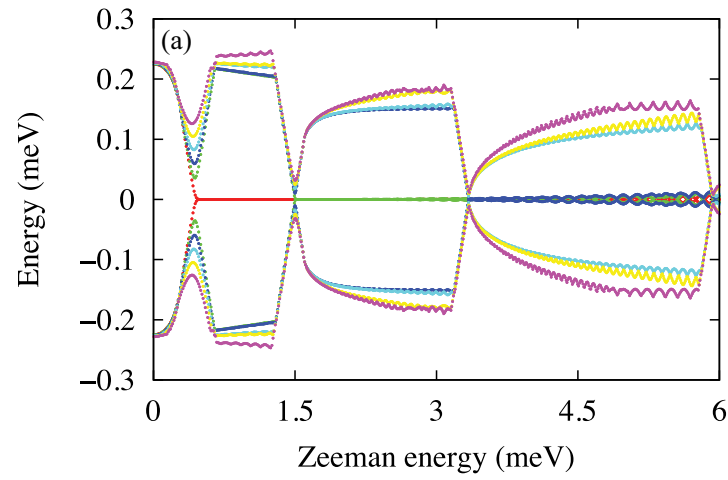

(b)
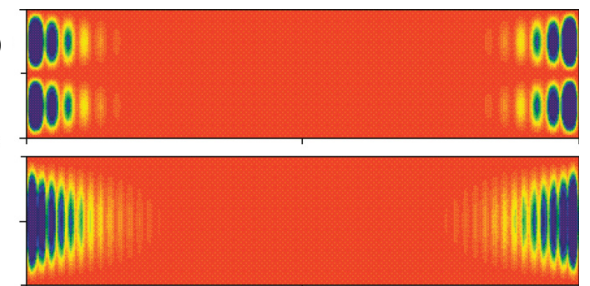

FIG. 2. (Color online) Results neglecting Rashba mixing, $\alpha_{y}=0$ in Eq. (4), and without magnetic orbital effects, for field orientation along $x$. (a) Spectrum of eigenvalues as a function of Zeeman energy $\Delta_{B} \equiv g \mu_{B} B / 2$. Only the twelve eigenvalues closer to zero are displayed; higher or lower eigenvalues than the ones displayed correspond to bulk excitations and are not shown. Parameters: $L_{y}=$ $150 \mathrm{~nm}, L_{x}=3 \mu \mathrm{m}, \Delta=0.225 \mathrm{meV}, \mu=0, \alpha_{x}=0.045 \mathrm{eVnm}$. (b) Probability density for the Majorana-like edge modes corresponding to the second and first transverse modes for a Zeeman energy of $2 \mathrm{meV}$ in panel (a).

large Zeeman energies at magnetic fields that exceed the critical value $B_{c}$ given by $g \mu_{B} B_{c} / 2=\sqrt{\mu^{2}+\Delta^{2}} \cdot{ }^{13,14}$ In the quasi-1D case one has a similar condition for the critical field $B_{c}\left(n_{y}\right)$ at which a Majorana pair emerges from the $n_{y}$ transverse mode: $g \mu_{B} B_{c}\left(n_{y}\right) / 2=\sqrt{\left(\varepsilon_{n_{y}}-\mu\right)^{2}+\Delta^{2}}$, where $\varepsilon_{n_{y}}$ is the transverse mode energy. This scenario is confirmed in Fig. 2(a) where we show the spectrum when the Rashba mixing term is absent and the magnetic field is applied along the $\hat{x}$ direction. Remarkably, by increasing the magnetic field successive gapped low-energy pairs, each belonging to a different transverse mode, appear in the spectrum. We emphasize that Fig. 2(a) corresponds to a situation in which transverse modes are uncoupled because $\alpha_{y}=0$. To better illustrate the edge character of the Majorana pairs we have plotted in Fig. 2(b) the probability density for the the first and second transverse modes for $\Delta_{B}=2 \mathrm{meV}$. Remarkably, the pair energies of the spectrum are not exactly zero, but oscillate around zero with increasing $B$, showing a steadily increasing amplitude. This is a finite-size effect, indicating that the shortness of the wire eventually dominates removing Majorana pairs from zero energy and destroying the gap with the nearby states.

The value of the Rashba coupling assumed in Fig. 2 is within the range of typical values for InAs-based nanostructures. ${ }^{36,37}$ In the present context, however, this coupling could be affected by the nearby superconductor. In first approximation we have neglected this variation. Anyway, we have also checked that 

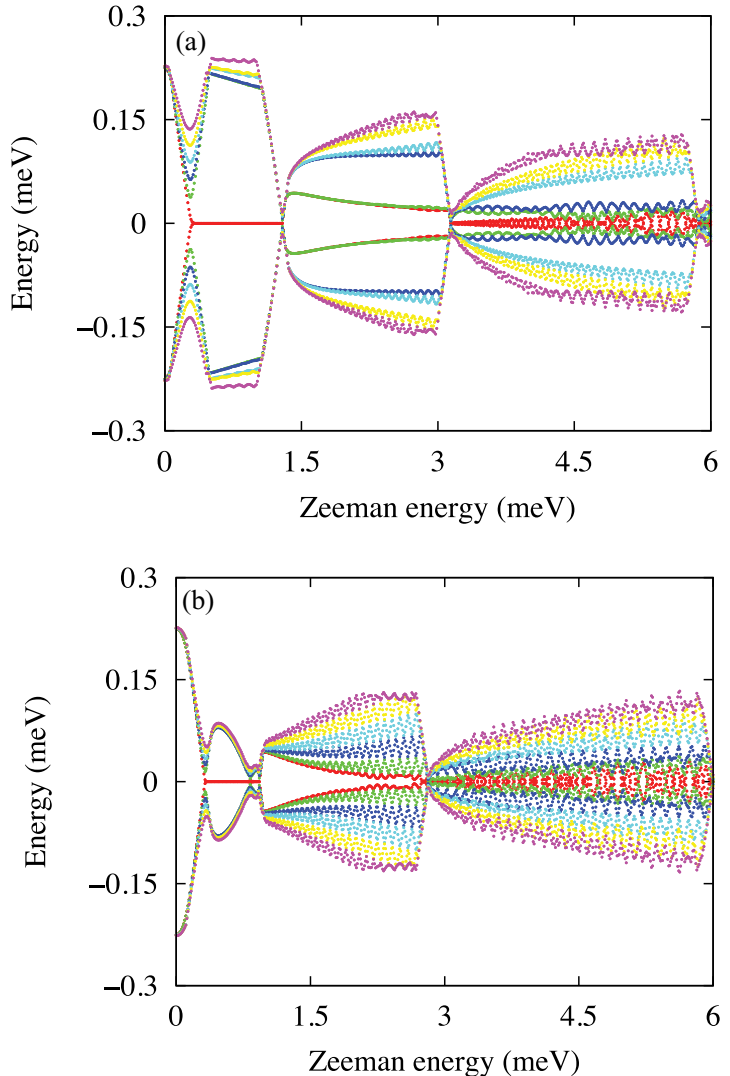

(c)
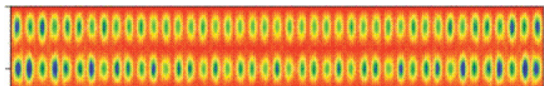
001000 00000000060000000000000000000010

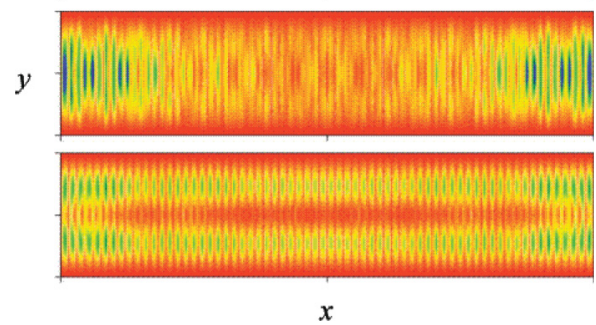

FIG. 3. (Color online) Same as Fig. 2 including Rashba mixing. The same parameters have been used, except for $\alpha_{y}=0.6 \alpha_{x}$ in panel (a) and $\alpha_{y}=\alpha_{x}$ in panel (b). The densities of panel (c) are for the three lowest modes when $\Delta_{B}=4 \mathrm{meV}$.

the physics we describe remains unaffected when $\alpha$ is modified by a factor 2 .

The effect of the Rashba mixing on the occurrence of multiple Majorana pairs is shown in Fig. 3. When Rashba mixing is present we find a single pair of Majorana modes in the low magnetic field regime. The Rashba mixing destroys the coexistence among different Majorana pairs at moderate magnetic fields. This is clearly illustrated in Fig. 3 where the spectrum is shown for $\alpha_{y}=0.6 \alpha_{x}$ [Fig. 3(a)], and $\alpha_{y}=\alpha_{x}$ [Fig. 3(b)]. In both cases, the Rashba mixing leads to an effective coupling between low-energy modes and dramatically affects the spectrum in the regions where two or three Majorana pairs would coexist. Because of the Rashba mixing, a clear gap region around zero energy appears [see Fig. 3(b) for $1 \mathrm{meV} \gtrsim \Delta_{B} \gtrsim 3 \mathrm{meV}$. By further increasing the magnetic field, there are no visible Majorana pairs of the spectrum in both Figs. 3(a) and 3(b). In these cases the eigenvalue spectrum becomes dense, trivial in topological language, due to the Rashba mixing. Therefore, the effect of Rashba mixing on the occurrence of multiple Majorana modes is twofold: Their zero-energy character is lifted and their gap protection from the rest of the states tends to vanish. Finally in Fig. 3(c) we show the spatial probability distributions of the three lowest eigenstates at large magnetic fields $\left(\Delta_{B}=4 \mathrm{meV}\right)$. In this case the Rashba mixing clearly destroys the edge-mode character of the states.

For completeness, we have investigated the robustness of the single Majorana regime when the chemical potential is changed (not shown here). We find an alternating trivial and nontrivial behavior as $\mu$ increases ${ }^{26}$ due to a sequence of occupied and unoccupied single transverse modes. Notice that single Majorana pairs do not necessarily emerge from the $n_{y}=1$ transverse mode but actually from the lowest-energy occupied transverse mode. A good strategy towards detecting Majorana modes is to use magnetic fields close to the critical field when only a single transversal mode contributes to the formation of a Majorana pair. In such situations, the sequence of occupied and unoccupied modes, as one increases the chemical potential, will produce a sequence of regions with a single Majorana pair each, so that Rashba mixing is not efficient. We emphasize that we do not find such alternating behavior between trivial/nontrivial phases when we vary the Zeeman field because of the Rashba mixing and the size effects. Nevertheless, the sequence of trivial/nontrivial phases
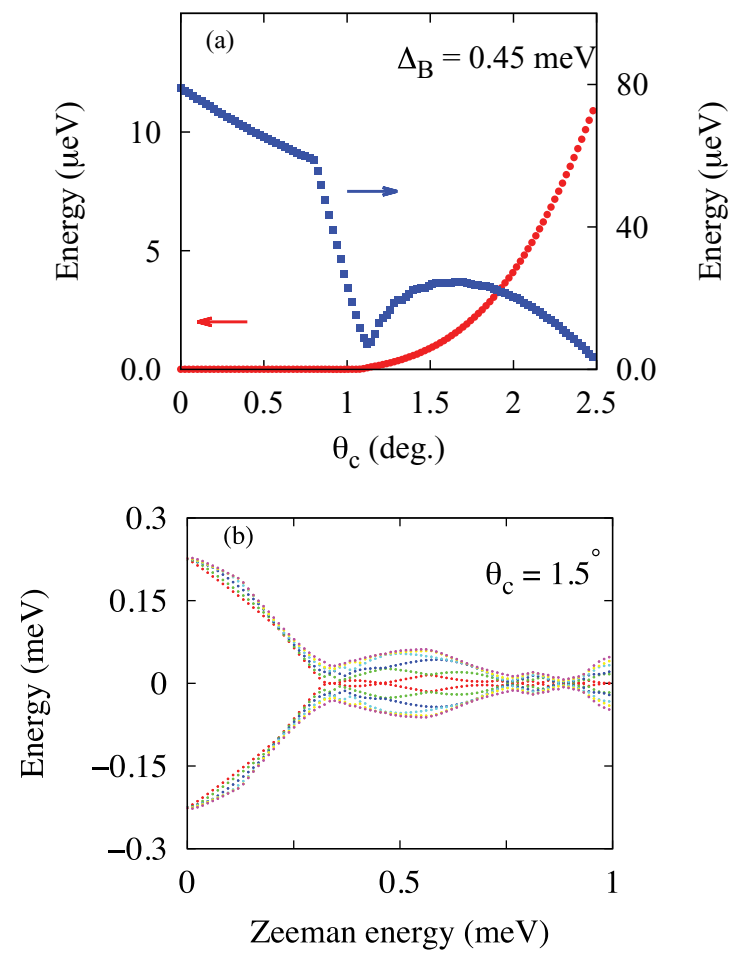

FIG. 4. (Color online) (a) Energy of the lowest eigenvalue as a function of the field tilting angle (circles) and of the gap with the next state (squares). We have used a Zeeman energy of $0.45 \mathrm{meV}$. (b) Same as Fig. 2 for a field tilting angle of $1^{\circ}$ out of the plane. Same parameters as Fig. 3(b). 
in the whole $\left(\mu, \Delta_{B}\right)$ phase space ${ }^{25-28}$ should be recovered for $L_{x} / L_{y} \rightarrow \infty$, when topological arguments become exact. From this point of view, our results demonstrate that the extrapolation of the topological phase diagram to finite samples with $L_{x} / L_{y} \approx 20$ is not sufficiently justified.

Magnetic orbital effects. All the above results were obtained assuming a magnetic field with a perfect in-plane orientation. We finish this discussion by studying the role of magnetic orbital effects, due to $B_{z} \neq 0$. The polar angle $\theta_{c}=\tan ^{-1} B_{z} / B_{x}$ quantifies a small out-of-plane deviation of the magnetic field. Our results are shown in Fig. 4. A small vertical component of the field is sufficient to steadily increase the energy of the lowest mode, from a clear Majorana-like character with $E_{1} \approx 0$ for $\theta_{c}=0^{\circ}$ to $E_{1} \approx 0.3 \Delta$ for $\theta_{c}=2.5^{\circ}$ [see Fig. 4(a)]. At the same time, the gap from the lowest to the next state, $E_{2}-E_{1}$, is reduced as the magnetic field deviation from the wire plane decreases. Thus, magnetic orbital effects suppress the Majorana character of the low-energy modes. This is demonstrated in Fig. 4(b), where just a few degrees of tilting are enough to destroy the low-energy modes as a function of $B$.

Conclusions. Summarizing, we have investigated the effect of the Rashba intermode coupling in finite multiband semiconductor nanowires when superconductivity is proximity induced and in the presence of a magnetic field. We find that Majorana physics appears provided that only one single transverse mode leads to the Majorana pair formation. The coexistence of multiple Majorana pairs is excluded by the presence of the Rashba mixing that hybridizes Majorana pairs and reduces their gap protection from the rest of the states. Additionally, we have studied how magnetic orbital effects affect the formation of gapped zero-energy modes. We conclude that even in the single Majorana mode regime, magnetic orbital effects lead to the destruction of those modes. While the fragility against magnetic orbital effects seems ultimately unavoidable in a planar geometry, the hybridization of multiple Majorana pairs could be eliminated as it has been shown in a recent proposal in Ref. 35, where Majorana physics occurs in nanowires without Rashba coupling. Here, Majorana modes originate by either the NW curvature or by an inhomogeneous Zeeman field generating effective banddiagonal coupling.

Note added. Recently, experimental evidence of Majorana modes in hybrid InSb nanowires has been presented. ${ }^{38,39}$ Although our geometry (planar) differs from the experimental one (cylindrical), we have computed Figs. 2 and 3 for the experimental values corresponding to InSb nanowires $(\Delta=$ $0.23 \mathrm{meV}$ and $\alpha=0.023 \mathrm{eV} \mathrm{nm}$ ) showing that the magnetic instability reported here [the splitting at large magnetic fields in Fig. 3(b)] agrees with the experiment. ${ }^{38}$ Nevertheless, the fragility predicted here against orbital effects has not been observed in the experiment when tilting the magnetic field in the vertical direction with respect to the cylinder axis. We believe this is due to the different role of tilting in planar and cylindrical geometries. A precise investigation of this mechanism is in progress.

Acknowledgments. We thank D. Sánchez for a critical reading of the manuscript and illuminating discussions. This work was supported by Grants No. FIS2008-00781, No. FIS2009-08744, No. FIS2011-23526, and No. CSD200700042 (CPAN) of the Spanish Government.
${ }^{1}$ P. M. Dirac, Proc. R. Soc. London, Ser. A 117, 610 (1928).

${ }^{2}$ E. Majorana, Nuovo Cimento 5, 171 (1937).

${ }^{3}$ G. Danby, J.-M. Gaillard, K. Goulianos, L. M. Lederman, N. Mistry, M. Schwartz, and J. Steinberger, Phys. Rev. Lett. 9, 36 (1962).

${ }^{4}$ F. Wilczek, Nat. Phys. 5, 614 (2009).

${ }^{5}$ N. B. Kopnin and M. M. Salomaa, Phys. Rev. B 44, 9667 (1991).

${ }^{6}$ G. Volovik, JETP Lett. 70, 609 (1999).

${ }^{7}$ N. Read and D. Green, Phys. Rev. B 61, 10267 (2000).

${ }^{8}$ G. Moore and N. Read, Nucl. Phys. B 360, 362 (1991).

${ }^{9}$ S. Das Sarma, C. Nayak, and S. Tewari, Phys. Rev. B 73, 220502 (2006).

${ }^{10}$ L. Fu and C. L. Kane, Phys. Rev. Lett. 100, 096407 (2008).

${ }^{11}$ L. Fu and C. L. Kane, Phys. Rev. B 79, 161408 (2009).

${ }^{12}$ J. Alicea, Phys. Rev. B 81, 125318 (2010).

${ }^{13}$ R. M. Lutchyn, J. D. Sau, and S. Das Sarma, Phys. Rev. Lett. 105, 077001 (2010).

${ }^{14}$ Y. Oreg, G. Refael, and F. von Oppen, Phys. Rev. Lett. 105, 177002 (2010).

${ }^{15}$ J. Linder and A. Sudbø, Phys. Rev. B 82, 085314 (2010).

${ }^{16}$ K. T. Law and P. A. Lee, Phys. Rev. B 84, 081304 (2011).

${ }^{17}$ L.-M. Duan, E. Demler, and M. D. Lukin, Phys. Rev. Lett. 91, 090402 (2003).

${ }^{18}$ C.-H. Cheng and S.-K. Yip, Phys. Rev. Lett. 95, 070404 (2005).

${ }^{19}$ S. Tewari, S. Das Sarma, C. Nayak, C. Zhang, and P. Zoller, Phys. Rev. Lett. 98, 010506 (2007).
${ }^{20}$ L. Jiang, T. Kitagawa, J. Alicea, A. R. Akhmerov, D. Pekker, G. Refael, J. I. Cirac, E. Demler, M. D. Lukin, and P. Zoller, Phys. Rev. Lett. 106, 220402 (2011).

${ }^{21}$ C. Nayak, S. H. Simon, A. Stern, M. Freedman, and S. Das Sarma, Rev. Mod. Phys. 80, 1083 (2008).

${ }^{22}$ S. Nadj-Perge, S. M. Frolov, E. P. A. M. Bakkers, and L. P. Kouwenhoven, Nature (London) 468, 1084 (2010).

${ }^{23}$ H. A. Nilsson, P. Caroff, C. Thelander, M. Larsson, J. B. Wagner, L. E. Wernersson, L. Samuelson, and H. Q. Xu, Nano Lett. 9, 3151 (2009).

${ }^{24}$ H. A. Nilsson, P. Samuelsson, P. Caroff, and H. Q. Xu, Nano Lett. 12, 228 (2012).

${ }^{25}$ R. M. Lutchyn, T. D. Stanescu, and S. Das Sarma, Phys. Rev. Lett. 106, 127001 (2011).

${ }^{26}$ A. C. Potter and P. A. Lee, Phys. Rev. B 83, 184520 (2011).

${ }^{27}$ T. D. Stanescu, R. M. Lutchyn, and S. Das Sarma, Phys. Rev. B 84, 144522 (2011).

${ }^{28}$ M. Gibertini, F. Taddei, M. Polini, and R. Fazio, Phys. Rev. B 85, 144525 (2012)

${ }^{29}$ R. M. Lutchyn and M. P. A. Fisher, Phys. Rev. B 84, 214528 (2011).

${ }^{30}$ A. Y. Kitaev, Phys. Usp. 44, 131 (2001).

${ }^{31}$ G. Kells, D. Meidan, and P.W. Brouwer, Phys. Rev. B 85, 060507 (2012).

${ }^{32}$ Ll. Serra, D. Sánchez, and R. López, Phys. Rev. B 72, 235309 (2005). 
${ }^{33}$ D. Sánchez and Ll. Serra, Phys. Rev. B 74, 153313 (2006).

${ }^{34}$ M. M. Gelabert, Ll. Serra, D. Sánchez, and R. López, Phys. Rev. B 81, 165317 (2010).

${ }^{35}$ M. Kjaergaard, K. Wölms, and K. Flensberg, Phys. Rev. B 85, 020503 (2012).

${ }^{36}$ P. Roulleau, T. Choi, S. Riedi, T. Heinzel, I. Shorubalko, T. Ihn, and K. Ensslin, Phys. Rev. B 81, 155449 (2010).
${ }^{37}$ S. Estévez Hernández, M. Akabori, K. Sladek, Ch. Volk, S. Alagha, H. Hardtdegen, M. G. Pala, N. Demarina, D. Grützmacher, and Th. Schäpers, Phys. Rev. B 82, 235303 (2010).

${ }^{38}$ V. Mourik, K. Zuo, S. M. Frolov, S. R. Plissard, E. P. A. M. Bakkers, and L. P. Kouwenhoven, Science 336, 1003 (2012).

${ }^{39}$ M. T. Deng, C. L. You, G. Y. Huang, M. Larsson, P. Caroff, and H. Q. Xu, arXiv:1204.4130. 